\title{
Tratamento cirúrgico das fraturas articulares desviadas do calcâneo por abordagem minimamente invasiva*
}

\author{
Surgical treatment of displaced intra-articular fractures \\ of the calcaneus using a less invasive approach
}

\author{
Fernando Araújo Silva Lopes'ㄹ, Roberto Zambelli de Almeida Pinto², \\ Eduardo Luiz Nogueira Gonçalves ${ }^{3}$, Gustavus Lemos Ribeiro Melo ${ }^{3}$, Renata Tavares dos Reis Leal ${ }^{3}$
}

\section{RESUMO}

Objetivos: Avaliar pacientes com fraturas articulares do calcâneo tratados entre dezembro de 2000 e agosto de 2007 por técnica cirúrgica minimamente invasiva, com ênfase nos resultados e complicações. Métodos: Estudo retrospectivo de 21 pacientes com 22 fraturas desviadas do calcâneo, tratadas com redução aberta ou fechada e fixação mínima. Nos 16 pacientes nos quais foi necessária a redução aberta, utilizou-se o acesso lateral direto e em nenhum placas ou enxerto ósseo. Resultados: O seguimento médio foi de três anos variando de seis a 82 meses. Foram empregados os métodos Maryland Foot Score e o escore de retropé da American Orthopaedic Foot and Ankle Society para avaliação clínica dos pacientes. Dezenove pés $(86,4 \%)$ tiveram resultados bons/ excelentes e três pés $(13,6 \%)$, resultados regular/

* Trabalho realizado no Serviço de Ortopedia e Traumatologia do Hospital Mater Dei - Belo Horizonte (MG), Brasil.

1. Médico Ortopedista e Cirurgião do Pé e Tornozelo, Chefe do Serviço de Ortopedia e Traumatologia do Hospital Mater Dei - Belo Horizonte (MG), Brasil.

2. Médico Ortopedista e Cirurgião do Pé e Tornozelo do Hospital Mater Dei - Belo Horizonte (MG), Brasil.

3. Residente (R3) em Ortopedia e Traumatologia do Hospital Mater Dei - Belo Horizonte (MG), Brasil.

Endereço para correspondência: Av. Olegário Maciel, 2.345, sala 501, Lourdes - 30180-112 - Belo Horizonte (MG), Brasil.

E-mail: fernandoaslopes@yahoo.com.br

Recebido em 3/6/08. Aprovado para publicação em 30/10/08.

Copyright RBO2008 ruim. Nenhum paciente apresentou complicação aguda da ferida cirúrgica, seja necrose de partes moles ou infecção. Conclusão: As técnicas cirúrgicas menos invasivas e fixações mínimas permitem resultados clínicos similares àqueles das técnicas mais agressivas, sem, entretanto, incidir em significativo número de complicações em partes moles e infecções.

Descritores - Calcâneo/lesões; Fraturas ósseas/cirurgia; Procedimentos cirúrgicos minimamente invasivos; Resultado de tratamento; Estudos retrospectivos

\section{ABSTRACT}

Objective: To evaluate patients with articular fractures of the calcaneus treated between December 2000 and August 2007 by minimally invasive surgical technique, with emphasis on results and complications. Methods: Retrospective study of 21 patients with 22 displaced fractures of the calcaneus, treated with open or closed reduction and minimal fixation. In the 16 patients for whom open reduction was required, right lateral access was used and plates or bone grafting were not used at all. Results: Mean follow-up was three years, varying from 6 to 82 months. The Maryland Foot Score and the hindfoot score of the American Orthopedic Foot and Ankle Society were used in the clinical evaluation of the patients. Nineteen feet (86.4\%) had good/excellent results and three feet (13.6\%) had regular/poor results. No patient had an acute complication of the 
surgical wound, either soft tissue necrosis or infection. Conclusion: Less invasive surgical techniques and minimal fixation allow for clinical results similar to those of more aggressive techniques, but without having a significant number of complications in soft tissues or infections.

\section{Keywords - Calcaneus/injuries; Fractures, bone/surgery; Surgical procedures, minimally invasive; Treatment outcome; Retrospective studies}

\section{INTRODUÇÃO}

As fraturas do calcâneo permanecem como um dos grandes desafios da traumatologia, seja pela morbidade e elevada incidência de resultados insatisfatórios, seja pela diversidade de filosofias e métodos de tratamento, ainda sem consenso na literatura.

A questão inicial é a compreensão da fisiopatologia da fratura, a visualização tridimensional dos componentes e sua relação com as articulações vizinhas. O não entendimento desses aspectos é o primeiro obstáculo ao procedimento mais adequado no tratamento das várias configurações que as fraturas apresentam ${ }^{(1)}$. Outro aspecto a considerar é a característica anatômica própria do retropé, sendo o calcâneo o osso de sustentação e amortecimento de cargas dinâmicas cíclicas, revestido por um estojo de partes moles vulnerável, com características especiais em relação aos outros segmentos do esqueleto.

A escolha da conduta terapêutica a adotar e a curva de aprendizado da técnica cirúrgica serão determinantes na evolução das fraturas do calcâneo ${ }^{(2)}$. Nos estudos retrospectivos de séries de pacientes tratados por diferentes métodos ${ }^{(1-7)}$, verificamos que a maioria dos autores concorda em que o tratamento conservador deve ser utilizado de maneira geral nas fraturas sem desvio ou pouco desviadas, assim como em pacientes idosos e com co-morbidades. Já as fraturas articulares desviadas, que acometem o adulto até a quinta ou sexta décadas da vida, terão melhores resultados se tratadas cirurgicamente, por qualquer dos métodos de redução e fixação conhecidos.

Qual dos caminhos seguir ao tratar as fraturas articulares desviadas do calcâneo? Para tal, o ortopedista deverá optar por método que entenda ser o de melhor custo-benefício para o paciente. Nas séries publicadas nos últimos 20 anos, a maioria dos autores preferiu a via de acesso lateral alargada e a osteossíntese com placa e parafusos ${ }^{(6,8-12)}$. Muitos desses pacientes evoluíram com complicações de partes moles entre 8,3\% e $32,8 \%$ dos casos, segundo Herscovici et al ${ }^{(12)}$. Além disso a necessidade de procedimentos complementares para retirada de implantes ocorre em cerca de 43,5\% dos pacientes, segundo Harvey et $a l^{(13)}$. Outros autores publicaram resultados utilizando métodos menos agressivos, com incisões menores e fixações com fios e parafusos, informando menor incidência de complicações de partes moles ${ }^{(3-4,14-16)}$.

Avaliando os resultados informados nas séries citadas, não encontramos diferenças significativas que justifiquem a opção pela via de acesso alargada e a fixação com placas, assim como o emprego de enxerto ósseo, considerado desnecessário pela maioria dos autores $^{(9)}$.

Baseados nesse conjunto de informações e conceitos e na própria experiência pessoal com os diversos métodos de tratamento, optamos, a partir do ano 2000, pela utilização da via de acesso lateral econômica e fixação mínima, tendo como premissa considerarmos inaceitável elevada incidência de complicações decorrentes do tratamento. Portanto, entendemos como minimamente invasiva a técnica que utiliza pequena incisão lateral, focada na articulação talocalcânea, para redução direta dos fragmentos articulares. Ela não promove nenhum deslocamento de partes moles e a fixação é feita apenas com fios e parafusos, não utilizando placa e enxerto ósseo.

O objetivo deste trabalho é avaliar os resultados funcionais obtidos no tratamento das fraturas desviadas do calcâneo por abordagem minimamente invasiva, com ênfase na incidência de complicações de partes moles, comparativamente aos métodos mais agressivos.

\section{MÉTODOS}

Foram tratados cirurgicamente no Serviço de Ortopedia e Traumatologia do Hospital Mater Dei de Belo Horizonte/MG, entre dezembro de 2000 e agosto de 




Gráfico 1 - Intervalo de avaliação x Quantidade (MFS)

2007, 25 pacientes (27 pés) com diagnóstico de fratura articular desviada do calcâneo. As fraturas foram classificadas pelo método radiográfico de Essex-Lopresti $^{(4)}$ e pela classificação tomográfica de Sanders $e t$ $a l^{(2)}$.

Não incluímos no estudo: dois pacientes tratados com artrodese subtalar primária, nos quais não se obteve, durante o procedimento, a recuperação satisfatória da anatomia do calcâneo; um paciente portador de seqüela de trauma raquimedular, devido à impossibilidade de avaliação funcional; e um paciente que não retornou para avaliação.

Trata-se, portanto, da análise retrospectiva de 21 pacientes (22 pés), nos quais se obteve a redução fechada em seis $(27,2 \%)$ e a redução aberta em 16 (72,8\%). Quanto à osteossíntese, em 10 pés $(45,4 \%)$ a fixação foi híbrida, associando parafusos e fios de Kirschner; em nove $(40,9 \%)$, usamos apenas parafusos; e em três $(13,6 \%)$, somente fios de Kirschner.

A abordagem utilizada foi o acesso lateral direto, restrito à superfície articular talocalcanear; não foram utilizadas placas e/ou enxerto ósseo. O tempo de espera pré-operatório obedeceu à regra de aguardar redução do edema e do pregueamento da pele (wrinkle sign $)^{(9)}$ por meio da elevação do membro e enfaixamento acolchoado, em domicílio, o que se obteve entre três e 16 dias (média de sete dias) após o trauma. Apenas um paciente, que apresentava sofrimento agudo de partes moles por pressão exercida por fragmento da tuberosidade posterior do calcâneo, teve tratamento cirúrgico imediato.

Todos os pacientes foram operados pelo mesmo cirurgião e a avaliação pós-operatória final realizada

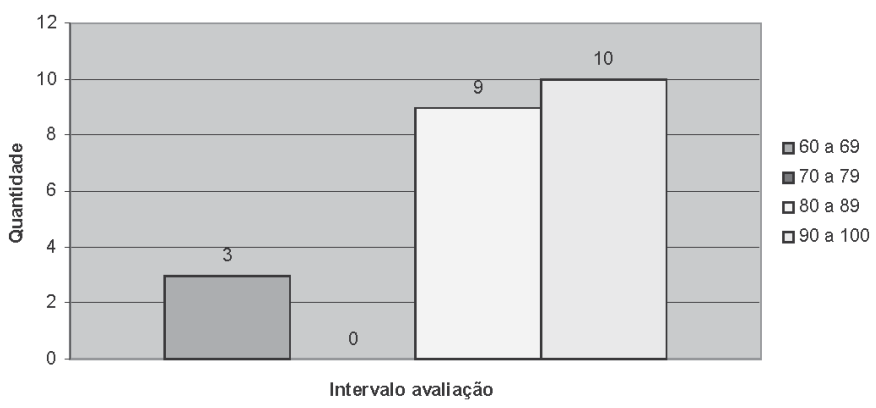

Gráfico 2 - Intervalo de avaliação x Quantidade (AOFAS)

pelos demais co-autores, utilizando-se o Maryland Foot Score (MFS) e o escore de retropé da American Orthopaedic Foot and Ankle Society (AOFAS) (gráficos $1 \mathrm{e}$ 2).

A adequada elaboração dos documentos de autorização e regulação de trabalho científico pela instituição não foi realizada, dada a natureza retrospectiva do estudo.

\section{Técnica cirúrgica}

Com o paciente em decúbito lateral, um fio de Steinmann de 4,0mm é introduzido no corpo do calcâneo para tração. Sob compressão manual, corrige-se o alinhamento axial e recuperam-se a altura e a largura. Associando a tração esquelética com movimentos de inversão e eversão e a compressão manual látero-lateral, verifica-se, através da fluoroscopia, a adequada recuperação da largura e altura do calcâneo. A redução aberta, quando necessária, é feita por acesso lateral, com incisão de aproximadamente $6 \mathrm{~cm}$, que se estende do ponto inframaleolar até a articulação calcaneocubóide. Os tendões fibulares e nervo sural são afastados distalmente, não se descolando as partes moles da parede lateral do calcâneo. Sob visão direta e auxílio da tração transesquelética, a parede lateral é afastada e reduzem-se os fragmentos articulares, usando como referência a superfície articular inferior do tálus. Em seguida, a parede lateral é fechada e fixada ao sustentáculo do tálus com dois fios-guia rosqueados, juntamente com os fragmentos articulares. Após confirmação fluoroscópica da adequada redução, procede-se à fixação com dois parafusos canulados. 
Obtida a fixação da superfície articular póstero-lateral, introduzem-se dois ou mais fios de Kirschner de $2,5 \mathrm{~mm}$, percutaneamente, desde a tuberosidade inferior, em direção ao tálus e ao cubóide, mantendo-se a correção do alinhamento axial e altura do calcâneo (nas fraturas pouco cominutivas, essa fixação não é necessária). O objetivo principal do procedimento é a recuperação da forma do calcâneo. Não se utiliza dreno em nenhum dos casos, mantendo o enfaixamento compressivo e a elevação do membro no pós-operatório. Em seis pacientes a redução foi fechada e efetuada fixação, percutânea, com fios e/ou parafusos canulados.

Algumas fraturas cominutivas ou do "tipo língua" tiveram a redução fechada e a fixação percutânea com fios e/ou parafusos, seja porque a cominuição impedia a fixação interna eficiente ou porque a redução do fragmento em língua foi obtida percutaneamente com manipulação tipo joystick.

A permanência hospitalar pós-operatória foi em média de 24 horas, os pontos retirados com 14 dias, os fios percutâneos, se utilizados, retirados entre cinco e seis semanas, quando é iniciada reabilitação fisioterápica. Inicia-se carga parcial progressiva após oito semanas e a carga total após 12 semanas.

\section{RESULTADOS}

Os pacientes foram avaliados com seguimento entre seis e 82 meses (média de três anos); apenas quatro pacientes tiveram seguimento inferior a um ano. Os avaliadores, residentes do serviço, participaram como auxiliares de cirurgia nos pacientes operados entre 2006 e 2007, e são co-autores. Os escores padronizados (retropé da AOFAS e Maryland Foot Score) escolhidos para a avaliação foram aplicados amplamente pelos co-autores em todos os pacientes convocados e efetivamente utilizados para coleta de dados.

Todos os pacientes avaliados foram chamados exclusivamente para a coleta de dados e informados do motivo de sua presença no serviço e da sua participação no trabalho em execução.

$\mathrm{Na}$ avaliação radiográfica, o ângulo de Böhler medido em 14 pacientes variou entre $3^{\circ}$ e $40^{\circ}$, média de $28^{\circ}$. Entretanto, o paciente que apresentou o ângulo de Böhler de $3^{\circ}$ teve resultado bom ( 89 e 82 pontos), enquanto dois dos três pacientes com resultados insatisfatórios tiveram ângulo de $40^{\circ}$. Essa avaliação por imagem foi padronizada com radiografias em: ânteroposterior e perfil do pé com apoio, oblíqua do pé e axial do calcâneo, todas realizadas no Serviço de Radiologia da instituição.

A avaliação clínica do alinhamento axial do calcâneo mostrou 19 pés $(86,4 \%)$ com alinhamento satisfatório e três pés $(13,6 \%)$ com alinhamento varo. Estes três pés apresentaram índices variados de resultado (um excelente, um bom e um regular).

Considerando o MFS, registramos 19 pés com resultados bons/excelentes $(86,4 \%)$ entre 84 e 100 pontos e três pés $(13,6 \%)$ com resultados regular/ruim, entre 49 e 74 pontos. Utilizando o escore AOFAS, observamos: 19 pés com resultados bons/excelentes $(86,4 \%)$ entre 82 e 100 pontos e três pés $(13,6 \%)$ com resultado regular, entre 62 e 63 pontos. Dos três pés com resultados insatisfatórios, dois não melhoraram após artrodese; um alcançou bom resultado após artrodese subtalar e correção de luxação dos tendões fibulares.

A média dos resultados segundo o Maryland Foot Score (MFS) foi 88,00000 e o desvio-padrão, 12,82044; o desvio-padrão foi aproximadamente $25 \%$, do tamanho do intervalo, em torno da média. Segundo o escore AOFAS, a média dos resultados foi 88,36364 e o desvio-padrão, 11,61717; o desvio-padrão foi aproximadamente $29 \%$, do tamanho do intervalo, em torno da média (tabela 1).

Não retornaram ao trabalho dois pacientes $(9 \%) \mathrm{e}$ cinco $(23,8 \%)$ não recuperaram a capacidade física prévia ao acidente. Considerando a mobilidade da articulação subtalar, oito pacientes (36,3\%) não apresentavam nenhuma perda na amplitude de movimento, oito $(36,3 \%)$ tiveram perda parcial e cinco $(22,7 \%)$, restrição importante da mobilidade da subtalar, sendo que três destes tiveram resultados satisfatórios segundo os questionários aplicados.

Pela classificação de Essex-Lopresti( ${ }^{(4)}, 14$ fraturas $(63,6 \%)$ eram do tipo depressão articular, cinco $(22,7 \%)$ do "tipo língua" e três $(13,6 \%)$ do tipo cominutiva indefinida. Os três pacientes com resultados insatisfatórios apresentavam fraturas do tipo depressão 
TABELA 1

Resultados baseados nos escores MFS e AOFAS

\begin{tabular}{|c|c|c|c|c|c|c|c|c|c|c|}
\hline Caso & Nome & Sexo & Idade & Lado & Mecanismo & Seguimento & $\mathbf{R X}$ & TC & Trat & $\begin{array}{c}\text { Resultados } \\
\text { (MFS / AOFAS) }\end{array}$ \\
\hline 1 & ALL & $M$ & 59 & D & Queda 5m & $44 m$ & $\mathrm{Cl}$ & IV & $R F+F K$ & $B / E$ \\
\hline 2 & ATAF & $M$ & 28 & $E$ & Queda & $6 m$ & LIN & IIC & $\mathrm{RF}+\mathrm{P}$ & $B / E$ \\
\hline 3 & ASN & $\mathrm{M}$ & 55 & $E$ & Queda 3,5m & $82 m$ & DA & IV & $R A+F K+P$ & $B / E$ \\
\hline 4 & AGM & $\mathrm{F}$ & 32 & $E$ & Acid. automob & $35 m$ & DA & III & $R A+F K+P$ & $B / E$ \\
\hline 5 & AAA & $M$ & 55 & $B-E$ & Queda $2 m$ & $30 \mathrm{~m}$ & & & $R F+P$ & $\mathrm{~B} / \mathrm{E}$ \\
\hline 5 & AAA & $\mathrm{M}$ & 55 & B-D & Queda 2m & $30 m$ & LIN & $\| A$ & $R A+P$ & $B / E$ \\
\hline 6 & CNFP & $\mathrm{F}$ & 28 & B-D & Queda 3m & $11 \mathrm{~m}$ & LIN & III & $\mathrm{RF}+\mathrm{P}$ & $B / E$ \\
\hline 7 & EVC & $M$ & 32 & $E$ & Queda 4m & $64 m$ & DA & III & $R A+F K+P$ & $B / E$ \\
\hline 8 & EMJV & $\mathrm{M}$ & 45 & $\mathrm{E}$ & Queda 3,5m & $79 m$ & $\mathrm{Cl}$ & IV & $\mathrm{RA}+\mathrm{FK}+\mathrm{P}$ & $\mathrm{B} / \mathrm{E}$ \\
\hline 9 & IS & $M$ & 54 & $E$ & Queda 2m & $18 m$ & DA & III & $R A+P$ & $B / E$ \\
\hline 10 & JNH & $\mathrm{F}$ & 13 & B-D & Queda 15m & $29 \mathrm{~m}$ & DA & IV & $R A+P$ & $B / E$ \\
\hline 11 & JAS & $\mathrm{M}$ & 59 & $\mathrm{E}$ & Queda $2 \mathrm{~m}$ & $66 \mathrm{~m}$ & DA & IV & $R A+P$ & $B / E$ \\
\hline 12 & JVP & $M$ & 52 & $\mathrm{D}$ & Queda 3m & $42 m$ & $\mathrm{Cl}$ & II & $\mathrm{RF}+\mathrm{FK}$ & $\mathrm{R} / \mathrm{R}$ \\
\hline 13 & MSSC & $\mathrm{F}$ & 40 & $\mathrm{D}$ & Acid. automob & $36 \mathrm{~m}$ & DA & IV & $R A+F K+P$ & $B / E$ \\
\hline 14 & MNBR & $\mathrm{F}$ & 62 & $\mathrm{D}$ & Acid. automob & $14 m$ & DA & & $R A+F K+P$ & $B / E$ \\
\hline 15 & MTC & $M$ & 40 & $E$ & Queda 1,70m & $9 m$ & DA & $\| A$ & $R A+F K+P$ & $B / E$ \\
\hline 16 & PRCC & $M$ & 38 & $\mathrm{D}$ & Queda 3,5m & $48 m$ & DA & IIIAB & $R A+P$ & $\mathrm{R} / \mathrm{R}$ \\
\hline 17 & RFF & $\mathrm{F}$ & 45 & $B-E$ & Queda 3m & $21 \mathrm{~m}$ & DA & II & $R A+F K+P$ & $\mathrm{~B} / \mathrm{E}$ \\
\hline 18 & SBM & $\mathrm{F}$ & 35 & $\mathrm{D}$ & Acid. automob & $12 m$ & $\mathrm{DA}$ & & $R A+P$ & $\mathrm{~B} / \mathrm{E}$ \\
\hline 19 & SMO & $\mathrm{F}$ & 50 & $\mathrm{D}$ & Queda 2m & $6 m$ & LIN & & $\mathrm{RF}+\mathrm{FK}$ & $\mathrm{B} / \mathrm{E}$ \\
\hline 20 & WCF & $\mathrm{F}$ & 26 & D & Queda $2 m$ & $30 \mathrm{~m}$ & LIN & III & $R A+F K+P$ & $\mathrm{~B} / \mathrm{E}$ \\
\hline 21 & ZMS & $\mathrm{F}$ & 74 & $\mathrm{D}$ & Acid. automob & $86 m$ & DA & IV & $R A+P$ & $\mathrm{~B} / \mathrm{E}$ \\
\hline
\end{tabular}

Legenda:

M - masculino; F- feminino; B - bilateral; D - direito; E - esquerdo; Cl - cominutiva indefinida; LIN - tipo língua; DA - tipo depressão articular; RA - redução aberta; RF - redução fechada; FK - fio Kirschner; P - parafuso.

MFS - Maryland Foot Score; AOFAS - American Orthopaedic Foot and Ankle Society; B/E - bom/excelente; R/R - ruim/regular. RX - Raios-X; TC - Tomografia computadorizada; Trat - Tratamento.

articular. Pela classificação tomográfica de Sanders ${ }^{(9)}$, sete $(31,8 \%)$ pés apresentavam fraturas do tipo II, oito do tipo III $(36,8 \%)$ e sete do tipo IV $(31,8 \%)$. Dos três resultados insatisfatórios, dois eram do tipo IV e um do tipo III; entretanto, cinco pacientes com fraturas do tipo IV tiveram resultados satisfatórios.

Considerando as complicações da ferida cirúrgica, nenhum paciente apresentou complicações agudas, tais como necrose de partes moles ou infecção. Um paciente evoluiu com neuroma do nervo sural, requerendo a amputação do coto neural associado à artrodese, sem, entretanto, melhorar o seu resultado, que foi insatisfatório. Uma paciente politraumatizada com fratura ipsilateral do fêmur apresentou artelhos em garra, porém seu resultado foi excelente em ambas as avaliações (98 e 100 pontos). Entre os pés, 12 (54,5\%) tiveram outras lesões associadas e, com exceção de um paciente que evoluiu com seqüela paralítica de trau- matismo raquimedular, em todos os outros não observamos interferência das lesões associadas no resultado final.

\section{DISCUSSÃO}

Em artigo de revisão das fraturas desviadas do calcâneo, Sanders afirmou que, embora a moderna abordagem cirúrgica tenha melhorado o resultado na maioria dos pacientes, não existe ainda um consenso quanto à classificação, tratamento e técnicas cirúrgicas a $\operatorname{adotar}^{(9)}$.

Essex-Lopresti publicou artigo fundamental ao entendimento do mecanismo, fisiopatologia e redução das fraturas do calcâneo, baseado em suas observações e de outros autores ${ }^{(4)}$. Entre as propostas para tratamento das fraturas de calcâneo destacam-se a de Bezes (205 fraturas $)^{(17)}$, Letournel (99 fraturas) $)^{(1)}$, Sanders et al (120

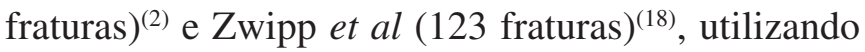


acesso lateral e a fixação com placas e parafusos. O que fica evidente nessas publicações é que os resultados dos tratamentos poderão ser melhores com procedimentos cirúrgicos, caso exista o bom entendimento da fisiopatologia, o recurso adequado de radiografias e tomografias e a fundamental curva de aprendizado a ser trilhada pelo cirurgião. Se estes aspectos não forem observados, os resultados do tratamento cirúrgico serão similares aos do tratamento conservador ${ }^{(9)}$.

Da mesma forma que observamos bons resultados com a técnica cirúrgica apurada, paralelamente estarão as complicações do método. Entre essas complicações estão as lesões neurais, as distrofias simpáticoreflexas, a artrite pós-traumática, a consolidação viciosa e as tendinites e luxações dos tendões fibulares ${ }^{(9)}$. As mais comuns, entretanto, são a deiscência da ferida cirúrgica e a necrose de partes moles, que eventualmente evoluem com infecção superficial ou profun$\mathrm{da}^{(12)}$. A presença da complicação em partes moles, ainda que evolua bem, requer curativos, desbridamentos e ocasionalmente retalhos, que atrasam a reabilitação, podendo resultar em rigidez, edema crônico e dor residual persistente. Observamos que, mesmo com a técnica adequada, os procedimentos mais agressivos, com incisões alargadas e fixação com placas e parafusos, a incidência de complicações de partes moles é elevada $(8,3 \text { a } 32,8 \%)^{(12)}$. A necessidade de cirurgias subseqüentes para retirada do implante chega a 43,5\% dos pacientes tratados ${ }^{(13)}$. Na nossa casuística não foram observadas: necrose de pele, deiscência de ferida, infecção de partes moles ou osteomielite. Estão publicados na literatura resultados de tratamentos cirúrgicos que avaliam técnicas, cujos princípios de menor agressão às partes moles e fixação mínima são empregados ${ }^{(3,15-}$ 16). Em relação aos bons resultados clínicos e funcionais, estes são similares àqueles em que se utilizam o acesso alargado e placas; entretanto, a incidência de complicações é significativamente menor. Deve-se levar em consideração que a fixação com placa não oferece a possibilidade de carga precoce e não acelera o tempo de reabilitação( ${ }^{(3)}$.

Neste trabalho publicamos os resultados obtidos em 16 pacientes portadores de fraturas articulares desviadas do calcâneo, tratados por método cirúrgico que teve como premissa a recuperação funcional possível, sem a incidência, que deve ser considerada como inaceitável, de complicações decorrentes do tratamento. A predominância de fraturas com depressão articular $(63,6 \%)$ e do tipo III $(36,8 \%)$ da classificação de Sanders $^{(9)}$ é concordante com a literatura. A avaliação radiográfica final do ângulo de Böhler, com média de $28^{\circ}$, reflete a adequada recuperação da forma anatômica do calcâneo com a técnica empregada. Conforme observado por Sanders, a redução articular anatômica, necessária para obter bom resultado, não assegura que isso irá ocorrer ${ }^{(9)}$. Na avaliação da mobilidade da articulação subtalar, observamos que $63,7 \%$ dos pacientes apresentavam alguma restrição de movimento, o que se demonstrou ser pouco significativo do ponto de vista funcional.

Os resultados avaliados pelos métodos MFS e AOFAS foram similares, tendo $86,4 \%$ dos pacientes apresentado resultados satisfatórios. Atribuímos esse índice, superior ao de algumas séries publicadas, ao perfil dos pacientes tratados no nosso serviço, usualmente de bom nível socioeconômico, sem restrições que comprometam o protocolo de tratamento, motivados na recuperação e, na maioria das vezes, independentes de possíveis ganhos secundários.

Nenhum paciente apresentou complicações agudas de feridas cirúrgicas, seja a necrose de partes moles ou infecções. Esse resultado confirmou o objetivo principal do método descrito, que foi o de obter o resultado médio satisfatório, comparável aos bons resultados da literatura, sem as complicações de partes moles e reintervenções comuns nos métodos mais agressivos de tratamento que utilizam vias de acesso alargadas e placas de sustentação.

\section{CONCLUSÃO}

As técnicas cirúrgicas menos invasivas e fixações mínimas permitem resultados clínicos similares àqueles das técnicas mais agressivas, sem, entretanto, incidir em significativo número de complicações em partes moles e infecções.

\section{REFERÊNCIAS}

1. Letournel E. Open treatment of acute calcaneal fractures. Clin Orthop Relat Res. 1993;(290):60-7. 
2. Sanders R, Fortin P, DiPasquale T, Walling A. Operative treatment in 120 displaced intraarticular calcaneal fractures. Results using a prognostic computed tomography scan classification. Clin Orthop Relat Res. 1993;(290):87-95.

3. Fernandez DL, Koella C. Combined percutaneous and "minimal" internal fixation for displaced articular fractures of the calcaneus. Clin Orthop Relat Res. 1993;(290):108-16.

4. Essex-Lopresti P. The mechanism, reduction technique and results in fractures of the os calcis. Br J Surg. 1952;39(157): 395-419.

5. Stephenson JR. Treatment of displaced intra-articular fractures of the calcaneus using medial and lateral approaches, internal fixation, and early motion. J Bone Joint Surg Am. 1987;69(1): 115-30.

6. Carr JB. Surgical treatment of the intra-articular calcaneus fracture. Orthop Clin North Am. 1994;25(4):665-75.

7. Kundel K, Funk E, Brutscher M, Bickel R. Calcaneal fractures: operative versus nonoperative treatment. J Trauma. 1996; 41(5):839-45.

8. Prado Júnior I, Rocha MA, Rezende RR. Tratamento cirúrgico das fraturas intra-articulares desviadas do calcâneo, através de osteossíntese interna, sem enxerto ósseo. Rev Bras Ortop. 1999; 34(7):421-9.

9. Sanders R. Displaced intra-articular fractures of the calcaneus. J Bone Joint Surg Am. 2000;82(2):225-50.

10. Murachovsky J, Martinelli MO, Ferreira RC, Fonseca Filho F. Fratura articular do calcâneo: resultado clínico-funcional do tratamento cirúrgico. Rev Bras Ortop. 2000;35(8):314-24.
11. Paul M, Peter R, Hoffmeyer P. Fractures of the calcaneum. A review of 70 patients. J Bone Joint Surg Br. 2004;86(8):1142-5.

12. Herscovici D Jr, Widmaier J, Scaduto JM, Sanders RW, Walling A. Operative treatment of calcaneal fractures in elderly patients. J Bone Joint Surg Am. 2005;87(6):1260-4.

13. Harvey EJ, Grujic L, Early JS, Benirschke SK, Sangeorzan BJ. Morbidity associated with ORIF of intra-articular calcaneus fractures using a lateral approach. Foot Ankle Int. 2001;22(11): 868-73.

14. Moraes Filho DC, Provenzano E, Mattos JR, Batista LC, Galbiatti JA, Ferreira JC, et al. Avaliação preliminar do tratamento cirúrgico de fraturas intra-articulares do calcâneo. Rev Bras Ortop. 1998;33(7):511-8.

15. Wiley WB, Norberg JD, Klonk CJ, Alexander IJ. "Smile" incision: an approach for open reduction and internal fixation of calcaneal fractures. Foot Ankle Int. 2005;26(8):590-2.

16. Ebraheim NA, Elgafy H, Sabry FF, Freih M, Abou-Chakra IS. Sinus tarsi approach with trans-articular fixation for displaced intra-articular fractures of the calcaneus. Foot Ankle Int. 2000; 21(2):105-13.

17. Bèzes H, Massart P, Delvaux D, Fourquet JP, Tazi F. The operative treatment of intraarticular calcaneal fractures. Indications, technique, and results in 257 cases. Clin Orthop Relat Res. 1993;(290):55-9.

18. Zwipp H, Tscherne H, Thermann H, Weber T. Osteosynthesis of displaced intraarticular fractures of the calcaneus. Results in 123 cases. Clin Orthop Relat Res. 1993;(290):76-86. 